\title{
Suppression of the long non-coding RNA MALAT-1 impairs the growth and migration of human tongue squamous cell carcinoma SCC4 cells
}

\author{
Xu Han, Zixiao Xu, Gang Tian, Zhen Tang, JianYong Gao, Yibo Wei, XiaoGang Xu
}

Department of Stomatology, Changhai Hospital Affiliated to Second Military Medical University, Shanghai, China

Submitted: 2 November 2016

Accepted: 6 June 2017

Arch Med Sci 2019; 15 (4): 992-1000

DOI: https://doi.org/10.5114/aoms.2018.73343

Copyright $\odot 2018$ Termedia \& Banach

\section{Abstract}

Introduction: Aberrant expression of long non-coding RNAs (IncRNAs) is associated with metastasis and poor prognosis in patients with various cancer types. However, few studies have assessed IncRNAs in oral squamous cell carcinoma (OSCC). This study aimed to investigate the expression and impact of IncRNAs in OSCC.

Material and methods: Real-time PCR analysis was used to examine the expression of four InCRNAs, MALAT-1, UCA1, BC200 and SRA, in 14 OSCC and adjacent normal tissue pairs. The impact of MALAT-1 suppression by SiRNA on the proliferation, apoptosis, anchorage-independent growth and migration of the human tongue carcinoma cell line SSC4 was also determined.

Results: MALAT-1 levels were significantly higher in the OSCC tissue than in the normal tissues $(p<0.004)$; no significant differences in UCA1, BC200 or SRA RNA levels were observed. Knockdown of MALAT-1 by siRNA significantly suppressed proliferation of SSC 4 cells $(p<0.004)$ and enhanced their apoptosis $(p<0.001)$. In addition, siRNA-mediated suppression of MALAT-1 inhibited SSC 4 cell colony formation $(p<0.001)$ and migration $(p<0.004)$. Conclusions: Elevated expression of MALAT-1 in OSCC may play a role in tumorigenesis and/or metastasis. Further studies are necessary to identify the mechanism by which MALAT-1 influences SCC4 growth and migration and validate its increased expression in OSCC patients.

Key words: oral squamous cell carcinoma, long non-coding RNA, MALAT-1, human tongue carcinoma cell line (SCC4).

\section{Introduction}

Long non-coding RNA (IncRNA) was first identified by Okazaki et al. [1] in 2002 when they conducted large-scale sequencing of mouse cDNA. IncRNAs are non-protein coding transcripts (ncRNA) longer than 200 nucleotides. Recent studies have revealed that IncRNA play important roles in cell development and metabolism [2], especially in miRNA regulation and protein encoding. Furthermore, a variety of cancers have abnormal expression of specific IncRNA, which can form ribonucleoprotein complexes and interact with proteins to alter the biobehavior of cancer cells [3]. Their putative role in the occurrence and development of cancers has also been suggested [4].

\author{
Corresponding authors: \\ XiaoGang Xu PhD \\ Department of Stomatology \\ Changhai Hospital \\ Affiliated to Second Military \\ Medical University \\ \#326 of No. 6 Building 3B \\ No. 168 \\ Changhai Road \\ Yangpu District Road \\ Shanghai, China \\ Phone: +86 021-31162031 \\ E-mail: xxgsmmu@sina.com \\ JianYong Gao \\ Department of Stomatology \\ Changhai Hospital \\ Second Military Medical \\ University \\ \#326 of No. 6 Building 3B No. \\ 168 Changhai Road, Yangpu \\ District Road, Shanghai, China \\ Phone: +86 021-31162031 \\ E-mail: yongjiangao1976@126. \\ com
}


Analysis of the IncRNA expression profile in normal oral mucosa and oral precancerous lesions revealed 325 differentially expressed IncRNA, $60 \%$ of which had abnormal expression in the oral precancerous lesions [5]. Metastasis-associated lung adenocarcinoma transcript 1 (MALAT-1) was the first IncRNA found to be related to cancer metastasis [5]. Ji et al. [6] found that MALAT-1 was highly expressed in non-small cell lung cancer (NSCLC) with early metastasis, and high MALAT-1 expression predicted a poor prognosis of NSCLC. In liver cancer, MALAT-1 played an important regulatory role in its occurrence and development [7], and it is upregulated in hepatocellular carcinoma (HCC), colon cancer, pancreatic cancer, breast cancer and prostate cancer [8-10]. Increased MALAT-1 expression in pharyngeal squamous cell carcinoma (SCC) was also demonstrated, increasing dramatically in stage T3 or T4 disease compared with stage T1 or T2 [11].

In addition to MALAT-1, roles for other InCRNA in cancer progression have been reported. Specifically, high UCA1 expression in bladder cancer cells increased their proliferation and resistance to therapies [12]. In breast cancer, UCA1 inhibited the tumor suppressor gene $P 27$ [13]; UCA1 expression was also correlated with lymph node metastasis in patients with tongue SCC (TSCC) [14]. In addition, another IncRNA, BC200, was overexpressed in lung cancer, breast cancer, cervical cancer and ovarian cancer [15]. Furthermore, steroid receptor RNA activator (SRA) may modulate androgen receptor and estrogen receptor activity; therefore, it may play an important role in breast cancer [16].

Because few studies have evaluated the expression of IncRNA in oral SCC (OSCC) [17], this study aimed to investigate the expression of MALAT-1, BC200, UCA1 and SRA in OSCC and examine their possible role in the occurrence and development of OSCC. These particular IncRNAs were selected because each has been shown to be differentially expressed in a wide variety of cancers, including oral cancers [18]. Specifically, MALAT-1 was overexpressed in OSCC, and its suppression in OSCC cell lines reduced epithelial mesenchymal transition, migration, and invasion as well as tumor growth in vivo [19]. Similarly, UCA1 levels were upregulated in tongue squamous cell carcinoma, and its silencing reduced OSCC proliferation and metastasis by altering WNT/ $\beta$-catenin signaling [20]. Although BC200 and SRA have not been reported in head and neck cancer, previous studies have shown that BC200 IncRNA is a potential predictive marker of poor prognosis in esophageal squamous cell carcinoma patients [21], and SRA is upregulated following vitamin $D$ receptor ablation in skin cancer [22]. In addition, IncRNA SRA variants are associated with breast cancer risk [23]. We first examined the expression profile of
MALAT-1, BC200, UCA1 and SRA in oral precancerous lesions by quantitative RT-PCR. Furthermore, the impact of MALAT-1 silencing on OSCC proliferation, apoptosis, colony formation capability and migration was examined in SCC4 cells.

\section{Material and methods}

\section{Collection of OSCC and adjacent normal tissues}

In total, 14 pairs of OSCC tissues and corresponding adjacent normal tissues were collected from inpatients who received surgical interventions in the Department of Stomatology at the Changhai Hospital. None of the patients received chemotherapy and radiotherapy before the surgery, and patients with recurrent OSCC were excluded. The age, gender, location of primary cancer, clinical stage and neck lymph node metastasis were recorded. This study was approved by the Ethics Committee of the Changhai Hospital, and informed consent was obtained from each patient before enrollment in the study.

The cancer tissues and adjacent normal tissues were collected during the surgery, and a fraction of each was processed for pathological examination to identify the cancer tissues and normal tissues under a light microscope. The remaining tissues were stored at $-80^{\circ} \mathrm{C}$ for further analysis.

\section{Quantitative RT-PCR}

Total RNA was isolated from frozen OSCC and adjacent normal tissues (about $100 \mathrm{mg}$ ) by the addition of $1 \mathrm{ml}$ of Trizol reagent (Invitrogen, Carlsbad, CA, USA). In addition to 1000 ng of RNA, the reverse transcription reaction $(20 \mu$ total) included the following: $4 \mu$ of $5 \times$ Primescript Buffer, $1 \mu$ l of PrimeScript RT Enzyme Mix, $1 \mu$ of $50 \mu \mathrm{M}$ OligodT Primer, $1 \mu$ l of $100 \mu \mathrm{M}$ random hexamers, and RNase-free deionized water (all from TaKaRa, Japan). The conditions for reverse transcription included $37^{\circ} \mathrm{C}$ for $15 \mathrm{~min}, 85^{\circ} \mathrm{C}$ for $5 \mathrm{~s}$, and $4^{\circ} \mathrm{C}$. Detection of MALAT-1, BC200, UCA1 and SRA expression was undertaken with an $A B I 7500$ real-time PCR instrument ( $A B I$, Foster City, CA, USA) and the primers shown in Table I (synthesized by Shanghai Sangon Biotech Co., Ltd, China). BRYT Green dye (Promega, Madison, WI USA) was used for the RT-PCR analysis, and the 20- $\mu \mathrm{l}$ reaction included the following: $10 \mu$ of Go Taq qPCR Master Mix, $0.4 \mu \mathrm{l}$ of forward primer $(10 \mu \mathrm{M})$ and $0.4 \mu \mathrm{l}$ of reverse primer $(10 \mu \mathrm{M}), 0.2 \mu \mathrm{l}$ of $100 \times \mathrm{CXR}$, $2 \mu \mathrm{l}$ of cDNA and $7 \mu \mathrm{l}$ of nuclease-free water. Detection was performed in triplicate, and $\beta$-actin served as an internal control. The conditions for PCR were as follows: predenaturation at $95^{\circ} \mathrm{C}$ for $2 \mathrm{~min}$, and then 40 cycles of $95^{\circ} \mathrm{C}$ for $15 \mathrm{~s}$ and $60^{\circ} \mathrm{C}$ for $1 \mathrm{~min}$. The relative expression of each gene was calculated using the $2^{-\Delta \Delta \mathrm{ct}}$ method. 
Table I. Primers used for the quantitative RT-PCR analysis

\begin{tabular}{|c|c|}
\hline Genes & Sequences $\left(5^{\prime}-3^{\prime}\right)$ \\
\hline \multirow[t]{2}{*}{ MALAT-1 } & GAATTGCGTCATTTAAAGCCTAGTT (sense) \\
\hline & $\begin{array}{l}\text { GTTTCATCCTACCACTCCCAATTAAT } \\
\text { (antisense) }\end{array}$ \\
\hline \multirow[t]{2}{*}{ UCA 1} & CTCTCCATTGGGTTCACCATTC (sense) \\
\hline & GCGGCAGGTCTTAAGAGATGAG (antisense) \\
\hline \multirow[t]{2}{*}{$B C 200$} & GGCCGGGCGCGGTGGCTCAC (sense) \\
\hline & AAAGGGGGGGGGGGGTTGTT (antisense) \\
\hline \multirow[t]{2}{*}{$S R A$} & TGGCTCTACTGGTGCAAGAGC (sense) \\
\hline & AACCATGAGGGAGCGGTG (antisense) \\
\hline \multirow[t]{2}{*}{$\beta$-Actin } & CGTGGACATCCGTAAAGACC (sense) \\
\hline & ACATCTGCTGGAAGGTGGAC (antisense) \\
\hline
\end{tabular}

\section{Cell culture and siMALAT-1 transfection}

We purchased SCC4 cells, a human tongue squamous cell carcinoma cell line derived from a 55-year-old man, from the ATCC (ATCC No. CRL1624; Manassas, VA, USA). SCC4 cells were maintained in $\alpha$-MEM containing $10 \%$ fetal bovine serum (FBS; both from GIBCO, Shanghai) at $37^{\circ} \mathrm{C}$ in an environment containing $5 \% \mathrm{CO}_{2}$ at $95 \%$ humidity.

Lipofectamine 2000 (Invitrogen) was used for the transfection of siMALAT-1 to inhibit MALAT-1 expression. Four pairs of MALAT-1-siRNA and a scrambled control were synthesized in Genepharma Biotech Co., Ltd. (China): MALAT-1siRNA-3097, 5'-GAGGUGUAAAGGGAUUUAUTT-3' and 5'-AUAAAUCCCUUUACACCUCTT-3'; MALAT-1SIRNA-5639, 5'-GGCAUUUGCAUCUUUAAAUTT-3' and 5'-AUUUAAAGAUGCAAAUGCCTT-3'; MALAT-1SiRNA-6108, 5'-CCCUCUAAAUAAGGAAUAATT-3' and 5'-UUAUUCCUUAUUUAGAGGGTT-3'; MALAT-1SIRNA-6960, 5'-GGCAAGUGGAAAUGUUUAATT-3' and 5'-UUAAACAUUUCCACUUGCCTT-3'; and Scrambled siRNA, 5'-UUCUCCGAACGUGUCACGUTT-3' and 5'-ACGUGACACGUUCGGAGAATT-3'.

After transfection of SCC4 cells with each siMALAT-1 pair as well as the scrambled control, MALAT-1 expression was detected by RT-PCR and compared with untransfected cells at 24 and $48 \mathrm{~h}$ after transfection. Briefly, the reverse transcription reaction included $2 \mu$ of RNA, $10 \mu$ of $2 \times$ reverse transcription buffer, $0.4 \mu \mathrm{l}$ of MMLV reverse transcriptase $(200 \mathrm{U} / \mu \mathrm{l}), 5 \mu \mathrm{l}$ of random primer N6 $(100 \mu \mathrm{M}), 3 \mu \mathrm{l}$ of dNTPs $(2.5 \mathrm{mM})$, and $0.15 \mu \mathrm{l}$ of RNasin $(40 \mathrm{U} / \mu \mathrm{l}$ ) (all from XX). The conditions for reverse transcription included $37^{\circ} \mathrm{C}$ for $60 \mathrm{~min}$, $85^{\circ} \mathrm{C}$ for $10 \mathrm{~min}$, and $4^{\circ} \mathrm{C}$. The $20-\mu l$ reaction for the quantitative RT-PCR included the following: $10 \mu \mathrm{l}$ of $2 \times$ quantitative PCR Master Mix, $0.08 \mu \mathrm{l}$ of forward primer $(20 \mu \mathrm{M})$ and $0.08 \mu \mathrm{l}$ of reverse primer $(20 \mu \mathrm{M}), 2 \mu \mathrm{l}$ of cDNA, $0.4 \mu \mathrm{l}$ of Taq DNA polymerase $(2.5 \mathrm{U} / \mu \mathrm{l})$ and nuclease-free water to $20 \mu \mathrm{l}$. The conditions for PCR were as follows: predenaturation at $95^{\circ} \mathrm{C}$ for $3 \mathrm{~min}$, and then 40 cycles of $95^{\circ} \mathrm{C}$ for $15 \mathrm{~s}$ and $62^{\circ} \mathrm{C}$ for $30 \mathrm{~s}$ and $72^{\circ} \mathrm{C}$ for $30 \mathrm{~s}$.

\section{Detection of SCC4 cell proliferation}

SCC4 cell proliferation was detected with a CCK-8 kit (Dojindo, Japan) following the manufacturer's instructions. Briefly, the cells were seeded into 96well plates at a density of $2 \times 10^{4}$ cells/well and transfected the following day as described above. At $24 \mathrm{~h}$ after transfection with scrambled siRNA and SiMALAT-1 6108 and 6960, cells were re-suspended in serum-free medium (GIBCO) and then incubated at $37^{\circ} \mathrm{C}$ in an environment with $5 \%$ $\mathrm{CO}_{2}$ for $24 \mathrm{~h}$. At 24,48 , and 72 h, $10 \mu \mathrm{l}$ of CCK-8 solution was added to each well, and cells were incubated for $1 \mathrm{~h}$. Absorbance was measured in a microplate reader at $450 \mathrm{~nm}$.

\section{Detection of SCC4 cell apoptosis}

Annexin V/PI double staining was performed to detect the apoptotic cells. After cell density was adjusted to $1 \times 10^{6} / \mathrm{ml}, 100 \mu \mathrm{l}$ of the cell suspension was mixed with $5 \mu$ l of Annexin V-FITC (mbchem). After the cells were incubated at room temperature in the dark for 5-15 $\mathrm{min}, 5 \mathrm{ml}$ of propidium iodide $(\mathrm{Pl})$ was added, and the cells were incubated in the dark at room temperature for 5-15 min. Following addition of $400 \mu \mathrm{l}$ of buffer, the cell suspension was subjected to flow cytometry at $480 \mathrm{~nm}$.

\section{Measurement of anchorage-independent growth of SCC4 cells}

For the lower gel, $1.2 \%$ agarose was mixed with $\alpha$-MEM containing $20 \%$ FBS at a ratio of $1: 1$, and $1.5 \mathrm{ml}$ of this mixture was added to each well of a 6-well plate, which was then allowed to stay at room temperature until the agarose solidified. For the upper gel, $0.7 \%$ agarose was mixed with $\alpha$-MEM containing $20 \%$ FBS at a ratio of $1: 1$, followed by addition of approximately 1000 transfected cells. This mixture was added to the pre-coated dishes. When the upper gel solidified, the dishes were incubated at $37^{\circ} \mathrm{C}$ in an environment with $5 \% \mathrm{CO}_{2}$. At 5, 6 and 7 days, three fields were randomly selected, the colonies were counted, and the average was calculated.

\section{Detection of SCC4 cell migration}

Cell migration was determined using Transwell chambers (pore size: $8 \mu \mathrm{m}$; Corning, Ithica, NY, USA). A cell suspension of approximately $1 \times 10^{5}$ cells in $100 \mu \mathrm{l}$ was added to the upper chamber of a Transwell chamber. Then, $500 \mu$ l of $\alpha$-MEM containing 20\% FBS was added to the lower chamber. 
After $48 \mathrm{~h}$, a swab was used to collect the cells in the upper chamber, and $4 \%$ paraformaldehyde was added to the lower chamber to fix the cells in the chamber for 20 min. After drying, crystal violet was added to the lower surface of the filter of the Transwell chamber, followed by incubation at room temperature for $30 \mathrm{~min}$. After washing in PBS once, cells were observed under an inverted microscope and photographed.

\section{Statistical analysis}

Data were presented as mean \pm standard deviation for normally distributed continuous variables or median (inter-quartile range, the range between the $25^{\text {th }}$ percentile and the $75^{\text {th }}$ percentile) for non-normally distributed scale parameters. The number and percentage were calculated for categorical variables. To examine the difference between groups, paired $t$-tests were carried out for comparisons between normal and cancer tissues; Wilcoxon signed rank tests were used when continuous variables had skewed distributions. A general linear model, including time, group, and interaction between these two parameters, was built to test whether the group differences were altered by time points or vice versa. Once a significant interaction between time and group was identified, the group difference at each time point and time effect within each group were separately examined by one-way analysis of variance (ANOVA). ANOVA was also used to compare between four or more groups. Bonferroni's method for variables with equal variances across groups or Dunnett's T3 test for parameters with unequal variances was then implemented after the ANOVA revealed significant findings. $P<0.05$ was considered significant. All statistics were two-sided, and the analyses were performed by SPSS software (version 15.0, SPSS Inc., Chicago, IL, USA).

\section{Results}

\section{OSCC patient characteristics}

A total of 14 patients were enrolled in this study. As shown in Table II, the average age of the study population was $55.1 \pm 17.0$ years (range: $25-$ 85 years). Most of the patients were male (64.3\%) with stage I OSCC (57.1\%). In addition, most of the samples were highly differentiated (71.4\%), and half of the patients had OSCC of the tongue (Table II).

\section{Expression of MALAT-1, BC200, UCA1 and SRA in OSCC and normal oral mucosa}

Because previous studies have reported putative roles for MALAT-1, BC200, UCA1 and SRA in
Table II. Demographic and clinical characteristics of the OSCC patients $(n=14)$

\begin{tabular}{|c|c|c|}
\hline Variable & $N$ & $\%$ \\
\hline Age, mean \pm SD [years] & $55.1 \pm 17$ & \\
\hline \multicolumn{3}{|l|}{ Gender: } \\
\hline Female & 5 & 35.7 \\
\hline Male & 9 & 64.3 \\
\hline \multicolumn{3}{|l|}{ Stage: } \\
\hline I & 8 & 57.1 \\
\hline II & 3 & 21.4 \\
\hline III & 3 & 21.4 \\
\hline \multicolumn{3}{|l|}{ Differentiation: } \\
\hline Moderate & 4 & 28.6 \\
\hline High & 10 & 71.4 \\
\hline \multicolumn{3}{|l|}{ Site: } \\
\hline Tongue & 7 & 50.0 \\
\hline Cheek & 4 & 28.6 \\
\hline Floor of the mouth or gums & 3 & 21.4 \\
\hline
\end{tabular}

other cancer types [5-17], we first analyzed their expression in 14 OSCC tissues and compared it with adjacent normal tissues. Compared with normal tissue, OSCC tissue showed a significantly increased MALAT-1 RNA expression ( $p=0.004$; Figure 1). However, no differences in BC200, UCA1 and SRA RNA expression were observed (Figure 1). The differential expression of MALAT- 1 between the OSCC and adjacent normal tissues suggests that it may have a role in OSCC progression. Thus, further studies focused on MALAT-1.

\section{The effects of MALAT-1 suppression on SCC4 cell proliferation and apoptosis}

To determine the impact of MALAT-1 in OSCC, we first suppressed its expression in SCC4 cells by siRNA. The inhibitory effects of four siRNAs (number 3097, 5639, 6108, and 6960) were first compared. As shown in Figure 2, only SiRNA 6960 significantly suppressed MALAT-1 RNA expression as compared to the untransfected, blank group ( $p=0.009)$. After $48 \mathrm{~h}$, both siRNA 6108 and 6960 significantly inhibited MALAT-1 gene expression as compared to the blank group (both $p \leq 0.016$; Figure 2). Therefore, subsequent experiments only used 6108 and 6960 siRNA.

We next determined the impact of MALAT-1 knockdown on SCC4 proliferation and apoptosis. Although no differences were seen at 24 and $48 \mathrm{~h}$, siRNA 6108 significantly inhibited SCC4 proliferation at $72 \mathrm{~h}$ as compared to the blank group ( $p=0.004$; Figure 3 ). In addition, silencing MALAT-1 expression with either 6108 or 6960 SiRNAs significantly increased the apoptosis rates of SCC 4 cells as compared to the blank group (both 
A

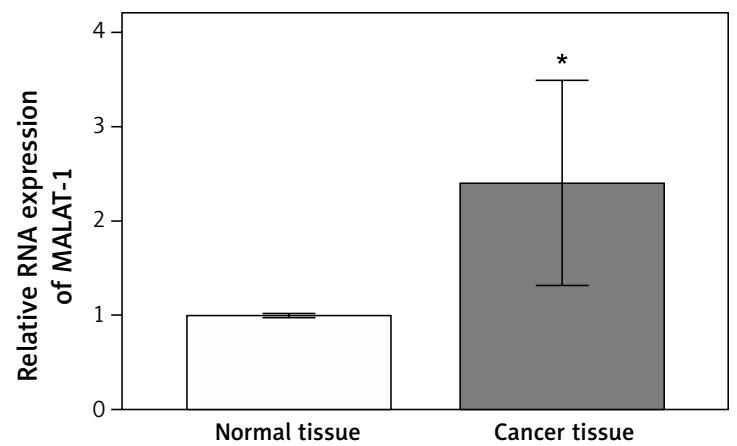

C

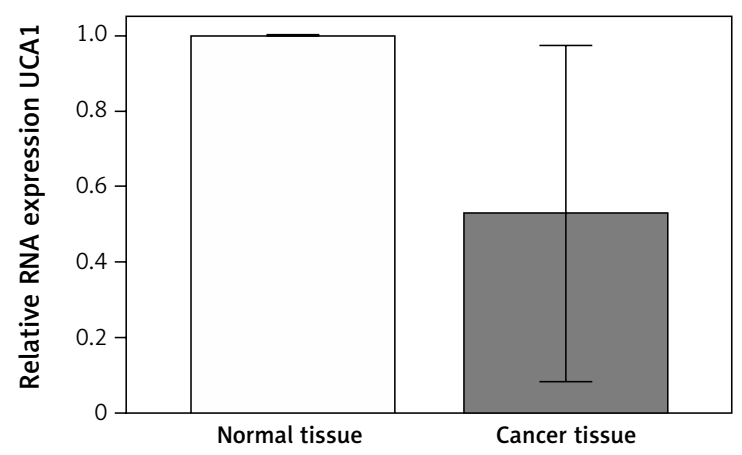

\section{B}

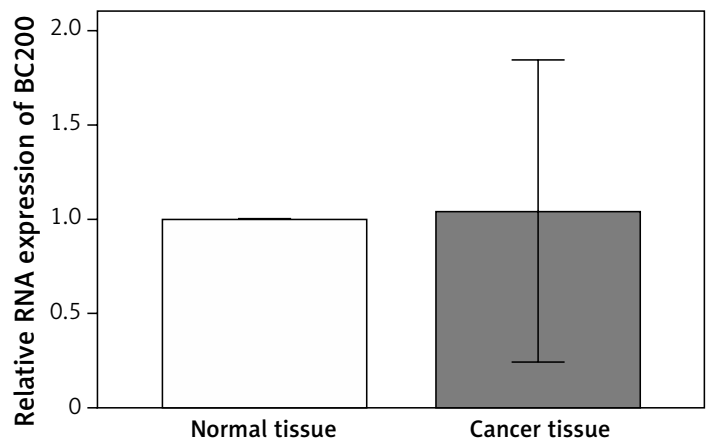

D

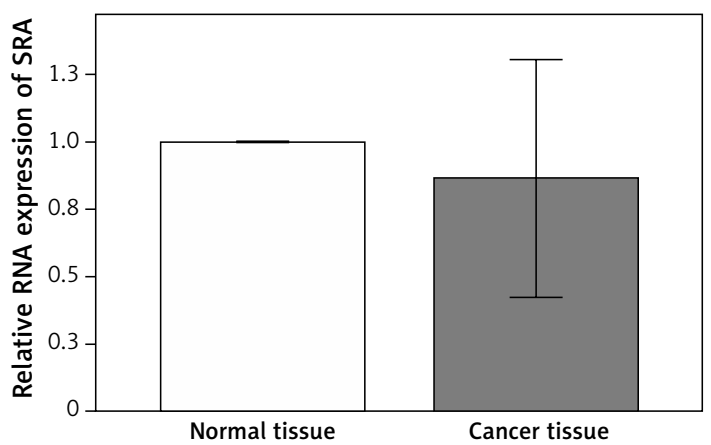

Figure 1. Expression of IncRNAs in patients with OSCC. MALAT-1 (A), BC200 (B), UCA1 (C), and SRA (D) expression was analyzed in OSCC and adjacent normal tissues by quantitative RT-PCR

Data were presented as mean \pm standard deviation and tested by paired $t$-test $\left(n=14\right.$ per group). ${ }^{*} p<0.05$.

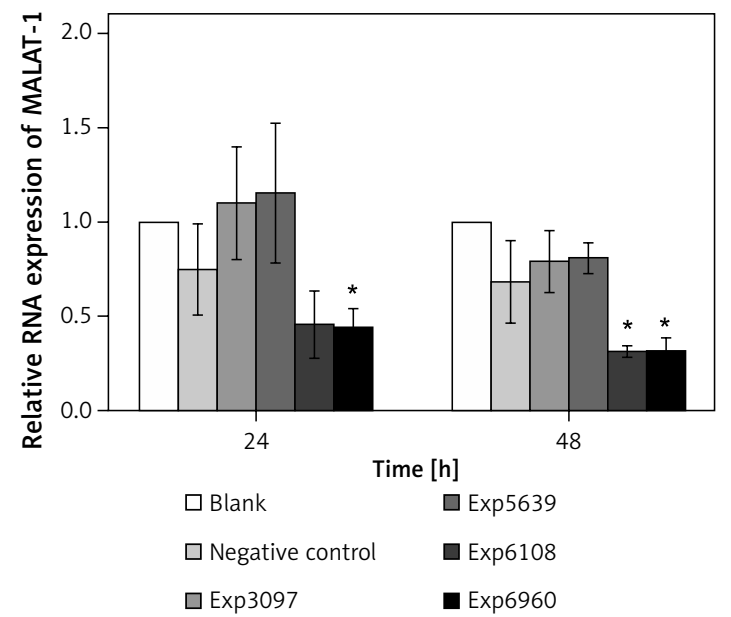

Figure 2. siRNA-mediated suppression of the MALAT-1 gene. The efficiency of MALAT-1 silencing was analyzed at 24 and $48 \mathrm{~h}$ following transfection in SCC4 cells by quantitative RT-PCR

Data were presented as mean \pm standard deviation and tested by analysis of variance ( $n=3$ per group). ${ }^{*} p<0.05$, significantly different from the blank group.

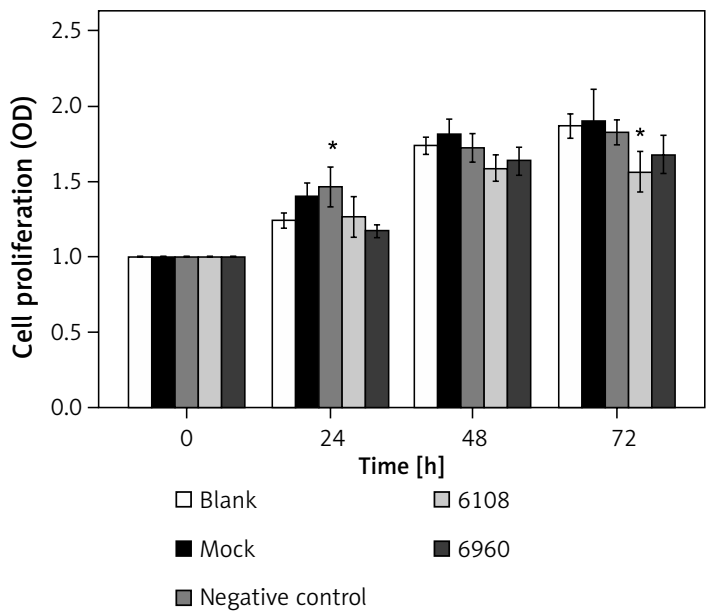

Figure 3. Effects of MALAT-1 knockdown on proliferation of SCC 4 cells ( $n=5$ per group). Data were presented as mean \pm standard deviation. Comparisons between groups at each time point or between time points within a given group are all tested by analysis of variance

${ }^{*} p<0.05$, significantly different from the blank group. 
A
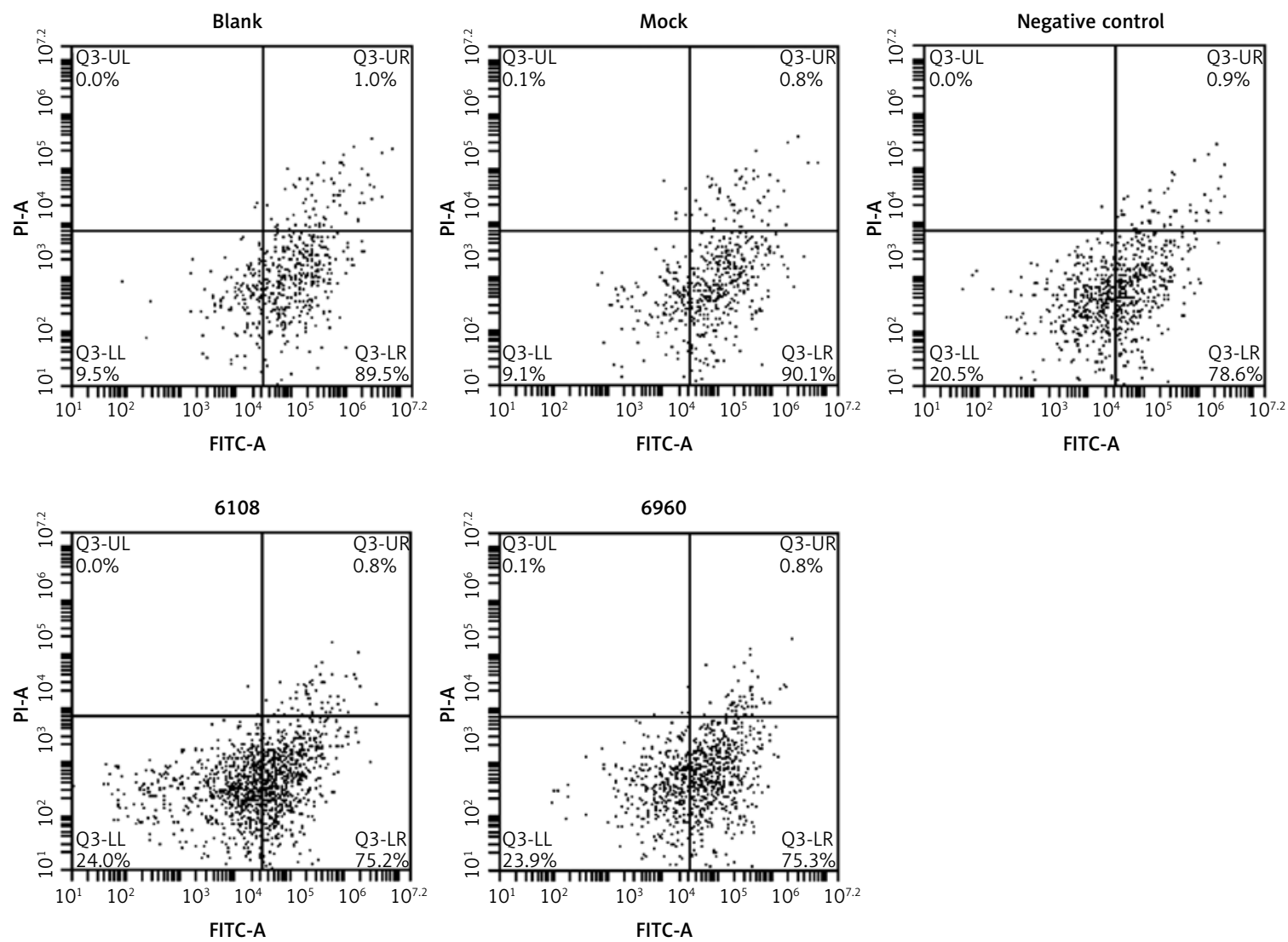

B

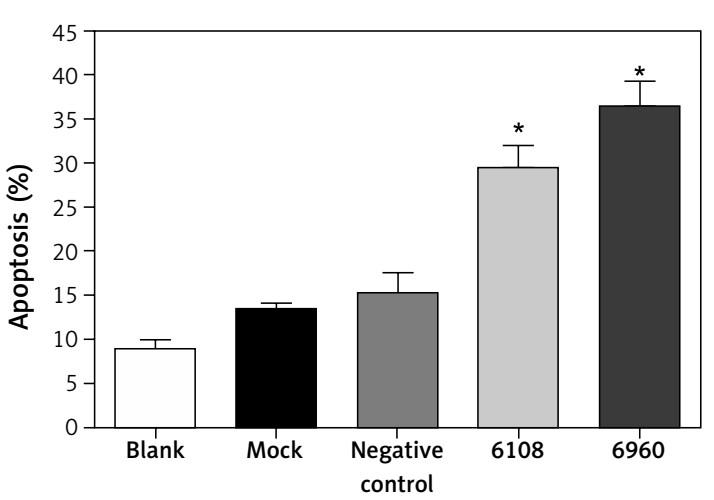

$p<0.001$; Figures 4 A and B). Thus, MALAT-1 expression alters SSC4 proliferation and apoptosis.

Inhibition of MALAT-1 expression reduces SCC4 cell anchorage-independent growth and migration

Previous studies on other cancer types have indicated a role for MALAT-1 in metastasis $[5,6]$. Therefore, we first examined the impact of MALAT-1 suppression on anchorage-independent growth using a soft agar colony formation assay (Figure $5 \mathrm{~A}$ ). As shown in Figure $5 \mathrm{~B}$, the number of clones was significantly reduced following si-
Figure 4. Effects of MALAT-1 knockdown on SCC4 cell apoptosis. A - Representative flow cytometry data from each group are shown. B - Apoptosis of SCC 4 cells ( $n=3$ per group). Data were presented as mean \pm standard deviation. Comparisons between groups at each time point or between time points within a given group are all tested by analysis of variance

${ }^{*} p<0.05$, significantly different from the blank group. 
A

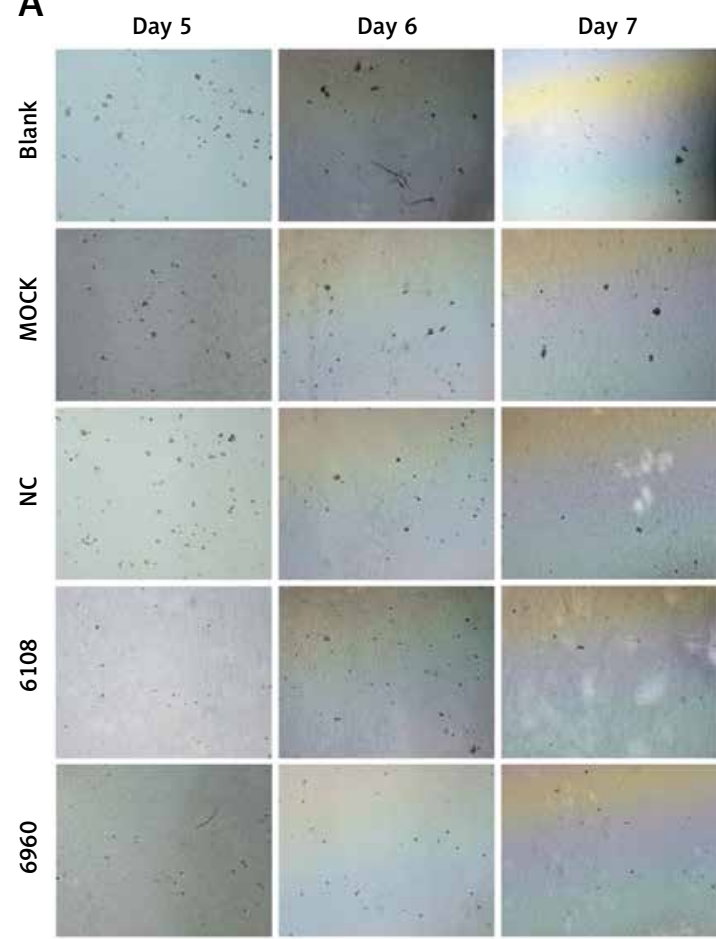

A

Blank

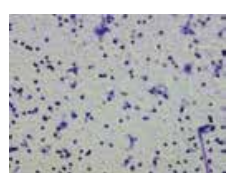

6108

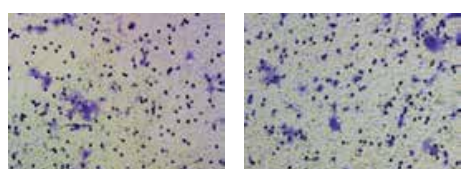

B

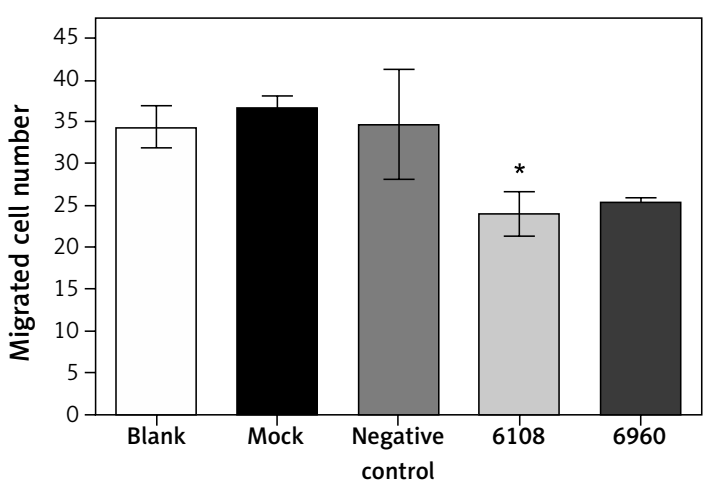

Figure 6. Effects of MALAT-1 knockdown on migration of SCC4 cells. A - Representative SCC 4 cell migration in each group. B - Migration of SCC4 cells using Transwell culture systems ( $n=3$ per group). Data were presented as mean \pm standard deviation. Comparisons between groups at each time point or between time points within a given group are all tested by analysis of variance. ${ }^{*} P<0.05$, significantly different from the blank group
B

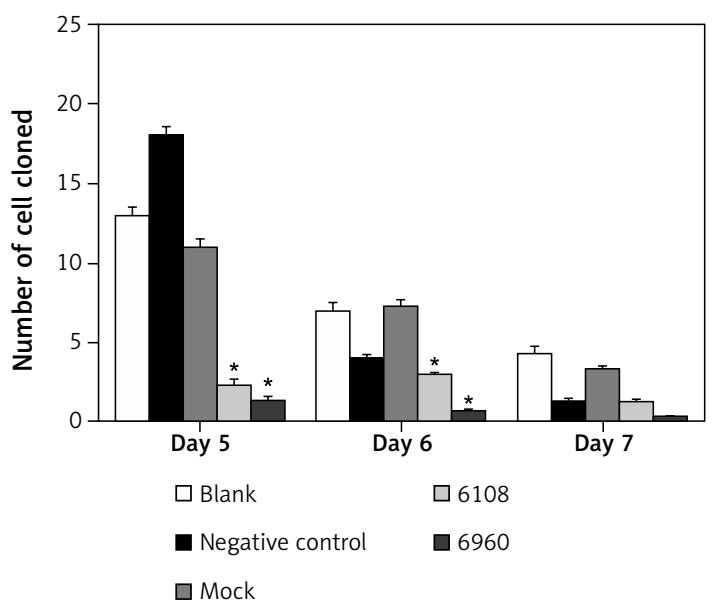

Figure 5. Effects of MALAT-1 knockdown on anchorage-independent growth of SCC4 cells. A - Representative SCC4 cell colony growth in each group at days 5-7. B - Anchorage-independent growth of SCC4 cells ( $n=3$ per group). Data were presented as mean \pm standard deviation. Comparisons between groups at each time point or between time points within a given group are all tested by analysis of variance. ${ }^{*} p<0.05$, significantly different from the blank group

\section{Discussion}

Although previous studies have shown a role for IncRNA in cancer progression and metastasis [24], few studies have assessed their expression and function in OSCC [17]. In the present study, MALAT-1 was differentially expressed between the OSCC and adjacent normal tissue, which is consistent with Fang et al. [14], who analyzed 94 TSCC samples and observed increased MALAT-1 expression. Furthermore, its suppression via siRNA resulted in increased SCC4 cell apoptosis as well as reduced proliferation, anchorage-independent growth, and migration. These studies suggest that increased MALAT-1 expression in OSCC may promote tumor cell growth and metastasis.

Analysis of two TCGA databases for head and neck cancer that included the four IncRNAs we analyzed in the present study (MALAT-1, UCA1, BC200 and SRA) revealed that MALAT-1 had a higher degree of gene amplification in 530 samples as compared with the other three IncRNAs. Consistent with our data showing a reduction in anchorage-independent growth and migration of SCC4 cells following MALAT-1 suppression, previous studies have suggested an association of MALAT-1 with metastasis and ultimately patient survival. Specifically, in patients with pharyngeal squamous cell carcinoma, increased MALAT-1 expression was observed in those with neck lymph node metastasis [11]. High MALAT-1 expression was also detected in nasopharyngeal carcinoma 5-8F cells with high metastatic potential [25]. 
Several studies have examined the mechanism by which MALAT-1 may promote tumor metastasis. In a manner similar to that described for the IncRNA HOTAIR, which decreases E-cadherin expression to promote OSCC metastasis [26], MALAT-1 overexpression in nasopharyngeal carcinoma CNE-1 cells by RNA activation inhibited epithelial cadherin and vimentin expression, which was accompanied by elevated invasion and metastasis [25]. In bladder cancer cells, MALAT-1 downregulation suppressed the expression of genes involved in epithelial-mesenchymal transition (EMT) [27]. In addition, the regulation of genes related to cell motility, including CTHRC1, CCT4, HMMR and $R O D 1$, by MALAT-1 has been shown [28], which is similar to recent studies in which IncRNAs regulated matrix metalloproteinase expression in OSCC $[29,30]$. Thus, further studies will examine the mechanism through which MALAT-1 promotes SSC4 cell migration.

In addition to impacting cell migration, MALAT-1 silencing reduced SSC4 cell proliferation and increased their apoptosis. This is consistent with data from CNE-1 cells in which MALAT-1 overexpression increased their proliferation [25]. However, the mechanism by which MALAT-1 influences cell growth and apoptosis remains unknown.

Once MALAT-1 is synthesized by RNA polymerase II, the products localize to nuclear speckles. Intranuclear MALAT-1 produces a short tRNAlike molecule, mascRNA, and a long MALAT-1 containing a poly $(A)$ tail, which localizes to the cytoplasm, suggesting that they possess distinct activities [31]. MALAT-1 is co-localized to nuclear speckles with the members of serine/arginine-rich (SR) splicing factors, regulating their nuclear localization [32]. Furthermore, MALAT-1 can bind to SR protein and regulate its phosphorylation, which then regulates the alternative splicing of pre-mRNA [33]. MALAT-1 may regulate chemokine receptor pre-mRNA processing by activation of SR protein, thereby affecting chemokine receptor expression in OSCC and impacting its migration. Thus, further studies are required to determine whether the effects of MALAT-1 suppression observed in the present study are mediated through its regulation of SR protein.

In the present study, the expression of UCA1, BC200, and SRA was comparable between OSCC and normal tissues. This is not consistent with previously reported studies describing UCA1 overexpression in bladder cancer [12], BC200 overexpression in lung cancer, breast cancer, cervical cancer and ovarian cancer [15]; and SRA overexpression in breast cancer [16]. This is likely due to specific differences between tumor types.

The present study has some limitations that warrant consideration. Firstly, the sample size used in this study was relatively small, and the correlation between MALAT-1 expression and patient clinical characteristics, including tumor stage recurrence, lymph node metastasis, and patient prognosis, was not analyzed. Furthermore, the impact of MALAT-1 overexpression on SCC4 cells was not determined. Also, the specific regulatory mechanisms by which MALAT-1 silencing altered SCC4 cell growth and migration were not determined, and will be examined in further studies by our group. Finally, MALAT-1 function was only analyzed in a single cell line; thus, further studies in other tongue squamous cell carcinoma cell lines are required to confirm these results.

In conclusion, MALAT-1 is highly expressed in OSCC as compared to adjacent normal tissue. Silencing the MALAT-1 gene inhibited SCC4 cell proliferation, anchorage-independent growth and migration and promoted apoptosis. Thus, MALAT-1 may play an important regulatory role in OSCC progression. Further studies with larger sample sizes are necessary to confirm these findings and correlate MALAT-1 expression with patient outcomes. In addition, studies to elucidate the mechanism through which MALAT-1 expression impacts SSC4 cell growth and migration are necessary.

\section{Acknowledgments}

$\mathrm{Xu}$ Han and Zixiao Xu contributed equally to this work.

\section{Conflict of interest}

The authors declare no conflict of interest.

\section{References}

1. Okazaki Y, Furuno M, Kasukawa T, et al. Analysis of the mouse transcriptome based on functional annotation of 60,770 full-length cDNAs. Nature 2002; 420: 563-73.

2. Ponting CP, Oliver PL, Reik W. Evolution and functions of long noncoding RNAs. Cell 2009; 136: 629-41.

3. Wang J, Zhang J, Zheng $\mathrm{H}$, et al. Mouse transcriptome: neutral evolution of 'non-coding' complementary DNAs. Nature 2004; 431: 1-757.

4. Su PF, Lee TC, Lin PJ, et al. Differential DNA methylation associated with hepatitis B virus infection in hepatocellular carcinoma. Int J Cancer 2007; 121: 1257-64.

5. Gibb EA, Enfield KS, Stewart GL, et al. Long non-coding RNAs are expressed in oral mucosa and altered in oral premalignant lesions. Oral Oncol 2011; 47: 1055-61.

6. Ji P, Diederichs S, Wang W, et al. MALAT-1, a novel noncoding RNA, and thymosin beta4 predict metastasis and survival in early-stage non-small cell lung cancer. Oncogene 2003; 22: 8031-41.

7. Lin R, Maeda S, Liu C, Karin M, Edgington TS. A large noncoding RNA is a marker for murine hepatocellular carcinomas and a spectrum of human carcinomas. Oncogene 2006; 26: 851-8.

8. Lai MC, Yang Z, Zhou L, et al. Long non-coding RNA MALAT-1 overexpression predicts tumor recurrence of 
hepatocellular carcinoma after liver transplantation. Med Oncol 2012; 29: 1810-6.

9. Luo JH, Ren B, Keryanov S, et al. Transcriptomic and genomic analysis of human hepatocellular carcinomas and hepatoblastomas. Hepatol 2006; 44: 1012-4.

10. Xu C, Yang M, Tian J, Wang X, Li Z. MALAT-1: a long non-coding RNA and its important 3'end functional motif in colorectal cancer metastasis. Int J Oncol 2011; 39: 169-75.

11. Feng J, Tian L, Sun Y, et al. Expression of long non-coding ribonucleic acid metastasis-associated lung adenocarcinoma transcript- 1 is correlated with progress and apoptosis of laryngeal squamous cell carcinoma. Head Neck Oncol 2012; 4: 46.

12. Matouk IJ, DeGroot N, Mezan S, et al. The H19 non-coding RNA is essential for human tumor growth. PLoS One 2007; 2: e845.

13. Huang J, Zhou N, Watabe K, et al. Long non-coding RNA UCA1 promotes breast tumor growth by suppression of p27 (Kip1). Cell Death Dis 2014; 5: e1008.

14. Fang Z, Wu L, Wang L, Yang Y, Meng Y, Yang H. Increased expression of the long non-coding RNA UCA1 in tongue squamous cell carcinomas: a possible correlation with cancer metastasis. Oral Surg Oral Med Oral Pathol Oral Radiol 2014; 117: 89-95.

15. lacoangeli A, Lin Y, Morley EJ, et al. BC200 RNA in invasive and preinvasive breast cancer. Carcinogenesis 2004; 25: 2125-33.

16. Leygue E. Steroid receptor RNA activator (SRA1): unusual bifaceted gene products with suspected relevance to breast cancer. Nucl Recept Signal 2007; 5: e006.

17. Yu T, Li C, Wang Z, et al. Non-coding RNAs deregulation in oral squamous cell carcinoma: advances and challenges. Clin Transl Oncol 2016; 18: 427-36.

18. Gibb EA, Brown CJ, Lam WL. The functional role of long non-coding RNA in human carcinomas. Mol Cancer 2011; 10: 38.

19. Zhou X, Liu S, Cai G, et al. Long non coding RNA MALAT1 promotes tumor growth and metastasis by inducing epithelial-mesenchymal transition in oral squamous cell carcinoma. Sci Rep 2015; 5: 15972.

20. Yang YT, Wang YF, Lai JY, et al. Long non-coding RNA UCA1 contributes to the progression of oral squamous cell carcinoma by regulating the WNT/ beta-catenin signaling pathway. Cancer Sci 2016; 107: 1581-9.

21. Zhao RH, Zhu CH, Li XK, et al. BC200 LncRNA a potential predictive marker of poor prognosis in esophageal squamous cell carcinoma patients. Onco Targets Ther 2016; 9: 2221-6.

22. Jiang YJ, Bikle DD. LncRNA profiling reveals new mechanism for VDR protection against skin cancer formation. I Steroid Biochem Mol Biol 2014; 144: 87-90.

23. Yan R, Wang K, Peng R, et al. Genetic variants in IncRNA SRA and risk of breast cancer. Oncotarget 2016; 7: 22486-96.

24. González-Ramírez I, Soto-Reyes E, Sánchez-Pérez Y, Herrera LA, García-Cuellar C. Histones and long non-coding RNAs: the new insights of epigenetic deregulation involved in oral cancer. Oral Oncol 2014; 50: 691-5.

25. Xie L, Hu Z, Wang X, Li Z. Expression of long noncoding RNA MALAT1 gene in human nasopharyngeal carcinoma cell lines and its biological significance. Nan Fang Yi Ke Da XueXueBao 2013; 33: 692-7.

26. Wu $Y$, Zhang $L$, Zhang $L$, et al. Long non-coding RNA HOTAIR promotes tumor cell invasion and metastasis by recruiting EZH2 and repressing E-cadherin in oral squamous cell carcinoma. Int J Oncol 2015; 46: 2586-94.
27. Ying L, Chen Q, Wang Y, Zhou Z, Huang Y, Qiu F. Upregulated MALAT-1 contributes to bladder cancer cell migration by inducing epithelial-to-mesenchymal transition. Mol Biosyst 2012; 8: 2289-94.

28. Tano K, Mizuno R, Okada T, et al. MALAT-1 enhances cell motility of lung adenocarcinoma cells by influencing the expression of motility-related genes. FEBS Lett 2010; 584: 4575-80.

29. Zhang S, Tian L, Ma P, et al. Potential role of differentially expressed IncRNAs in the pathogenesis of oral squamous cell carcinoma. Arch Oral Biol 2015; 60: 1581-7.

30. Kong XP, Yao J, Luo W, et al. The expression and functional role of a FOXC1 related mRNA-InCRNA pair in oralsquamous cell carcinoma. Mol Cell Biochem 2014; 394: 177-86.

31. Tripathi V, Ellis JD, Shen Z, et al. The nuclear-retained noncoding RNA MALAT1 regulates alternative splicing by modulating SR splicing factor phosphorylation. Mol Cell 2010; 39: 925-38.

32. Bernard D, Prasanth KV, Tripathi V, et al. A long nuclearretained non-coding RNA regulates synaptogenesis by modulating gene expression. EMBO J 2010; 29: 308293.

33. Fang $X$, Liu Z, Fan $Y$, et al. Switch to full-length of XAF1 mRNA expression in prostate cancer cells by the DNA methylation inhibitor. Int J Cancer 2006; 118: 2485-9. 